\title{
Optical scanning extrinsic Fabry-Perot interferometer for absolute microdisplacement measurement
}

\author{
Tianchu Li, Russell G. May, Anbo Wang, and Richard O. Claus
}

\begin{abstract}
We report an optical-scanning, dual-fiber, extrinsic Fabry-Perot interferometer system for absolute measurement of microdisplacement. The system involves two air-gapped Fabry-Perot cavities, formed by fiber end faces, functioning as sensing and reference elements. Taking the scanning wavelength as an interconverter to compare the gap length of the sensing head with the reference-cavity length yields the absolute measurement of the sensing-cavity length. The measurement is independent of the wavelength-scanning accuracy, and the reference-cavity length can be self-calibrated simply by one's changing the sensing-head length by an accurate value. (c) 1997 Optical Society of America
\end{abstract}

The sensing of displacement is generally the basis for measurements of various physical qualities, such as strain, pressure, vibration, and temperature. In 1983 Kersey et al. ${ }^{1}$ were among the first to demonstrate the use of an optical-fiber Fabry-Perot (FP) interferometer for measuring small displacement. In 1991 Murphy et al. ${ }^{2}$ proposed and demonstrated an extrinsic Fabry-Perot interferometric (EFPI) sensor. In this system two EFPI's were configured to produce two sets of output signals in quadrature phase so that bidirectional fringe counting could be obtained. The bidirectional fringe counting could improve the dynamic characteristic of the EFPI for measurements in a perturbation environment. Recently there has been a great interest in absolute displacement measurement by use of white-light fiber interferometers ${ }^{3,4}$ or optical-scanning EFPI's.5,6 In general, an unambiguous displacement measurement, which does not require a reference measurement to be made before the actual sensing procedure, is preferable in practical applications. In this paper we report an improved optical-scanning dual EFPI (DEFPI) sensing system for measuring microdis-

When this research was done all the authors were with the Fiber \& Electro-Optics Research Center, Bradley Department of Electrical Engineering, Virginia Polytechnic Institute and State University, Blacksburg, Virginia 24061-0111. T. Li is now with Length Division, National Institute of Metrology, Beijing 100013, China.

Received 21 February 1997; revised manuscript received 23 June 1997.

0003-6935/97/348858-04\$10.00/0

(C) 1997 Optical Society of America placement. The basic principle is to use a wavelength-tunable source to illuminate two EFPI's simultaneously, one as the sensing head and the other as the reference cavity. If the length of the reference cavity is precalibrated and remains constant, and the scanning wavelength is taken as an interconverter to compare the gap length of the sensing head with the reference-cavity length, an unambiguous measurement of the sensing-head length may thus be obtained.

For a standard EFPI the two beams, reflected from the two fiber ends in the sensing cavity, interfere with each other, and the signal received at the detector can be expressed as ${ }^{5}$

$$
\begin{aligned}
I_{(\lambda, L)} & =I_{o(\lambda)} \cos ^{2}\left[\left(\Phi_{s}+\Phi_{o}\right) / 2\right], \\
\Phi_{S} & =2 k n L=(4 \pi L n) / \lambda,
\end{aligned}
$$

where $\lambda$ is the free-space wavelength, $L$ is the FP cavity length, $I_{(\lambda, L)}$ is the intensity received at the detector as a function of $\lambda$ and $L, I_{o(\lambda)}$ represents the spectrum intensity, $\Phi_{s}$ is the phase difference between the two beams, $\Phi_{o}$ is the initial phase, $n$ is the refractive index of the medium filling the FP cavity, and $k=2 \pi / \lambda$.

If the wavelength is scanned from $\lambda_{1}$ to $\lambda_{2}$ while the cavity length remains constant, we have, from Eq. (2),

$$
\begin{aligned}
\Delta \Phi_{S} & =\int_{\lambda 1}^{\lambda 2} \mathrm{~d} \Phi_{S}=4 \pi L \int_{\lambda 1}^{\lambda 2}\left(-n / \lambda^{2}\right) \mathrm{d} \lambda \\
& =4 \pi L n \Delta \lambda /\left(\lambda_{1} \lambda_{2}\right),
\end{aligned}
$$




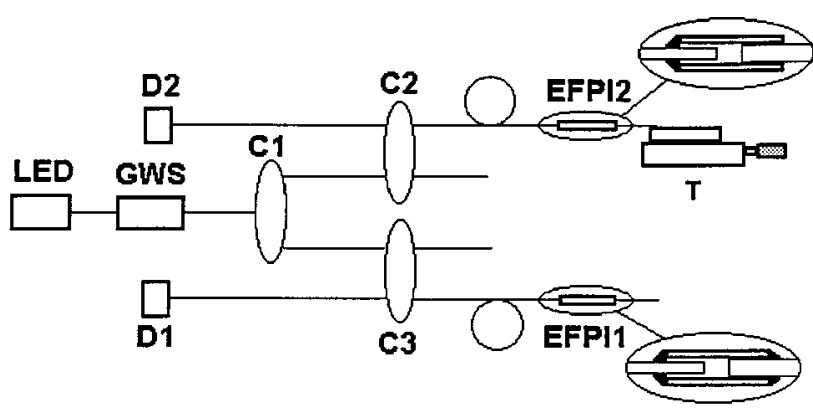

Fig. 1. Dual extrinsic FP interferometer (DEFPI). GWS, grating wavelength scanner; $\mathrm{C} 1, \mathrm{C} 2, \mathrm{C} 3$, couplers; $T$, translation stage; D1, D2, detectors.

where $\Delta \Phi_{S}$ is the phase change produced by the scanning of the wavelength from $\lambda_{1}$ to $\lambda_{2}$. Here we have assumed that the refractive index remains a constant. The unambiguous cavity length $L$ can then be calculated.

Equation (3) indicates that, to yield an accurate measurement of the FP cavity length, the wavelength scanning is required to be repeatable. In practice, accurate wavelength scanning is always difficult to achieve and maintain with either a monochromator, a CCD detector array-based optical spectrum analyzer, ${ }^{5}$ or with a tunable laser. ${ }^{6}$ Instead of measuring distance directly, it may be more practical to measure the FP length relative to the length of a fixed FP cavity by the wavelength-scanning method.

Figure 1 shows the experimental setup of the optical-scanning DEFPI. Two fiber EFPI's are connected to the output ports of two couplers C2 and C3. Each EFPI was constructed with two perpendicularly cleaved fiber ends inserted, face to face, into a silica hollow tube, forming a low-finesse FP cavity. ${ }^{1}$ The input-output fiber in the sensing EFPI was epoxied to the hollow tube end, and the sensing fiber was allowed to move freely inside the hollow tube. The sensing fiber was attached to a translation stage $T$ to provide accurate displacement for the experiments and for the self-calibration of the reference-cavity length (detailed as follows). For the reference EFPI, both fibers were epoxied to the two ends of the hollow tube to maintain a stable air cavity. The Fresnel reflections from the fiber-air interface (reference surface) and the reflection from the air-fiber interface (sensing surface) in each EFPI interfere in the input-output fiber. The interference signal is detected by the photo diodes $\mathrm{D}_{1}$ and $D_{2}$. The light from the tunable LED-grating assembly source [grating wavelength scanner (GWS), details given as follows] is split into two beams by coupler C1 to illuminate EFPI1 and EFPI2 through C2 and C3.

Recently, tunable optical-fiber lasers have been reported, ${ }^{7}$ and tunable laser diodes have become commercially available. Their performance is ideal to support the wavelength-scanning DEFPI reported in this paper, but their cost would prohibit most practical applications. We thus developed the LED-gratingGalvo scanner spectrum scanning assembly illustrated in Fig. 2. The light source is a 1300-nm edge-emitting

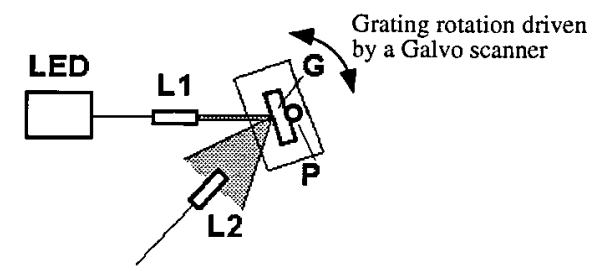

Fig. 2. Grating wavelength scanner (GWS). L1, L2, GRIN lens; G, grating, $P$, Galvo scanner shaft.

LED with full-width at half-power of $\sim 60 \mathrm{~nm}$. A $21^{\circ} 01^{\prime}$ blazed, 600 -line $/ \mathrm{mm}$ diffraction grating $\mathrm{G}$, as an optical-dispersion element, was mounted on the shaft $\mathrm{P}$ of a Galvo scanner constituting the GWS. The broadband spectrum light from the LED is collimated by a graded index (GRIN) lens, L1, and diffracted by the grating at the blaze angle. Part of the diffracted light is collected by another GRIN, L2, and focused into the receiving fiber. For a given grating, the spectral width of the light focused into the receiving fiber. For a given grating, the spectral width of the light focused into the receiving fiber is related to the distance between the grating and the fiber as well as to the numerical aperture of the GRIN lens-fiber assembly. In the experiment the spectral width was measured to be $6.3 \mathrm{~nm}$. When a precisely generated triangular driving current with a period of $0.2 \mathrm{~s}$ is applied to the Galvo, it turns the grating and hence tunes the wavelength of the light coupled into the receiving fiber. The wavelength-scanning range was $58 \mathrm{~nm}$.

From each EFPI a group of fringes is produced when the wavelength is scanned from $\lambda_{1}$ to $\lambda_{2}$ according to Eqs. (1)-(3). Using subscript 1 and 2 to denote the reference and the sensing EFPI's, we obtain from Eq. (3), by dividing the phase changes of these two independent EFPI's,

$$
\Delta \Phi_{S} 2 / \Delta \Phi_{S 1}=L_{2} / L_{1}
$$

What is interesting and perhaps more significant for the measurement is that Eq. (4) indicates that the measurement of sensing-cavity length $L_{2}$ is independent of wavelength $\lambda_{1}, \lambda_{2}$, and $\Delta \lambda$. That means we do not need the light source to be tuned accurately or repeatably.

In practice, the phase information can be derived from an analysis of the corresponding interference fringes. The fringe counting produced during one optical tuning cycle for each cavity is given as

$$
m_{i}=\Delta \Phi_{s i} / 2 \pi=\varepsilon_{i}+f_{i, 1}+f_{i, 2},
$$

where $m_{i}$ is the number of the fringes ( $i=1$ for the reference cavity and $i=2$ for the sensing head), $\varepsilon_{i}$ is the integer part of $m_{i}$, and $f_{i 1}$ and $f_{i 2}$ are the fractions before the first fringe peak and after the last peak, respectively.

The absolute gap length, $L_{2}$, of the sensing EFPI is derived as

$$
L_{2}=L_{1} m_{2} / m_{1}=L_{1}\left(\varepsilon_{2}+f_{2,1}+f_{2,2}\right) /\left(\varepsilon_{1}+f_{1,1}+f_{1,2}\right) .
$$


The first and the last fractions of the fringes can be repeatedly interpolated, or subdivided, to further improve the measurement resolution, either by electronics or by computer software. For example, if we assume the approximate linearity of the wavelength scanning versus time and denote the opticalscanning time for a fraction, for example, $f_{2,1}$, as $t_{2,1}$ and the scanning time for its first adjacent complete fringe $e_{2,1}$ as $t_{2,1,0}$, we thus have approximately

$$
f_{2,1} / e_{2,1}=t_{2,1} / t_{2,1,0} \text {. }
$$

The corresponding time is taken to subdivide the fringe fraction.

For the absolute FP cavity-length measurement, the reference-cavity length $L_{1}$ has to be accurately calibrated. This can be done by using an accurate displacement actuator (like the translation stage in Fig. 1) to change the gap length in the sensing head by a known distance. With the two sets of fringes, before and after the calibration displacement, counted for each EFPI and the accurate value of this displacement, from Eq. (6) we have

$$
\begin{aligned}
L_{1} & =\left(L_{2}{ }^{\prime}-L_{2}\right) /\left(m_{2}{ }^{\prime} / m_{1}{ }^{\prime}-m_{2} / m_{1}\right) \\
& =\Delta L_{2} /\left(m_{2}{ }^{\prime} / m_{1}{ }^{\prime}-m_{2} / m_{1}\right),
\end{aligned}
$$

where the prime superscript denotes the values after the calibration, whereas the values without the prime represent the values before the calibration displacement, and $\Delta L_{2}=L_{2}^{\prime}-L_{2}$.

The experimental fringes from the opticalscanning DEFPI are shown in Fig. 3. The lower trace in each figure is the driving current applied to the Galvo scanner, and the upper trace represents the interference fringes. Figure 3(a) is the fringes from the reference EFPI with a cavity length of 81.4 $\mu \mathrm{m}$, which was calibrated by the above self-contained procedure with an accuracy of $1 \mu \mathrm{m}$, limited by the accuracy of the translation stage (Fig. 1). The upper fringe curves in Figs. 3(b) and 3(c) are from the sensing head, and the cavity lengths are derived to be 106.1 and $148.3 \mu \mathrm{m}$, respectively.

The experimental curves in Fig. 3 prove the prediction of Eqs. (3) and (6) that the fringe number or the fringe frequency is proportional to the cavity length. The results shown in Figs. 3(b) and 3(c) were obtained from the sensing channel shown in Fig. 1 with different cavity lengths. These two curves show that the amplitude of the fringes decreases rapidly with the increase of the cavity length owing to the divergence of the light beam from the fiber end in the EFPI sensors. ${ }^{1,3}$

From 10 measurement data the repeatability is determined to be $0.2 \mu \mathrm{m}$, which is mainly limited by the low signal-to-noise ratio of the fringes, partially because of the low output power from the LED GWS. Consider the reference-cavity calibration accuracy of 1 $\mu \mathrm{m}$ mentioned above; the whole measurement accuracy of the sensing length was estimated to be $1 \mu \mathrm{m}$. If the new economic tunable laser diode's, or the next generation of tunable fiber lasers ${ }^{7}$ could be applied, the

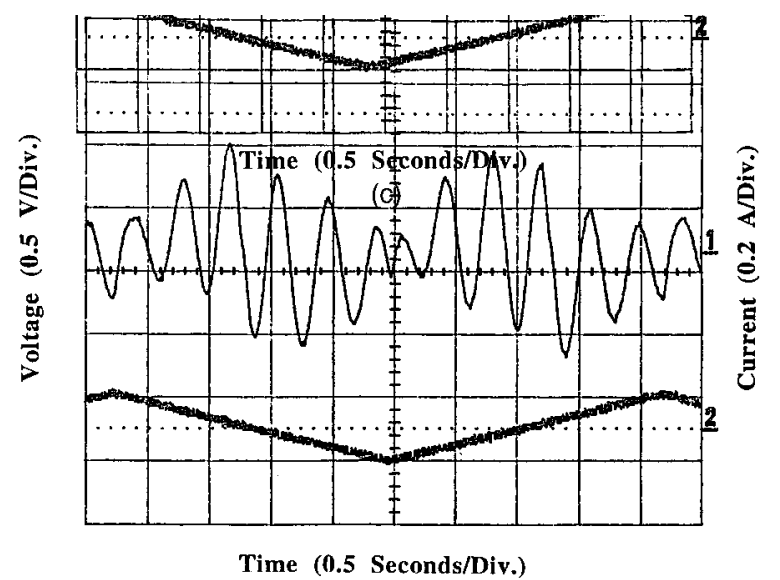

(a)

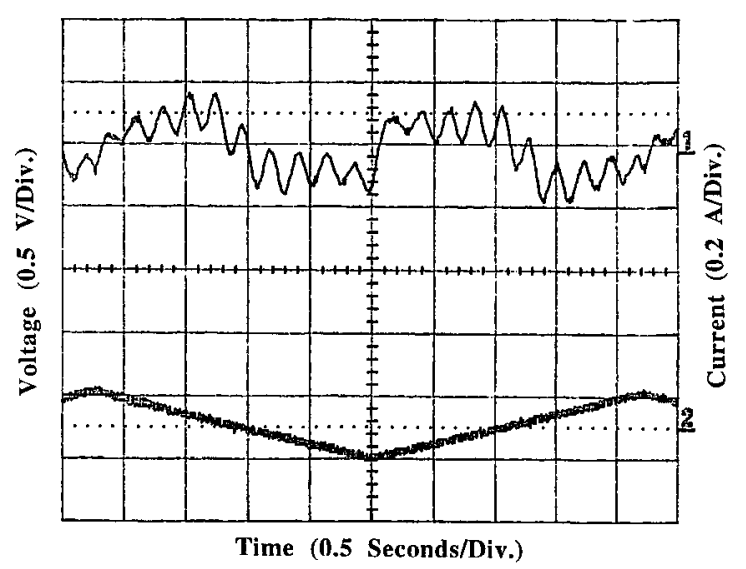

(b)

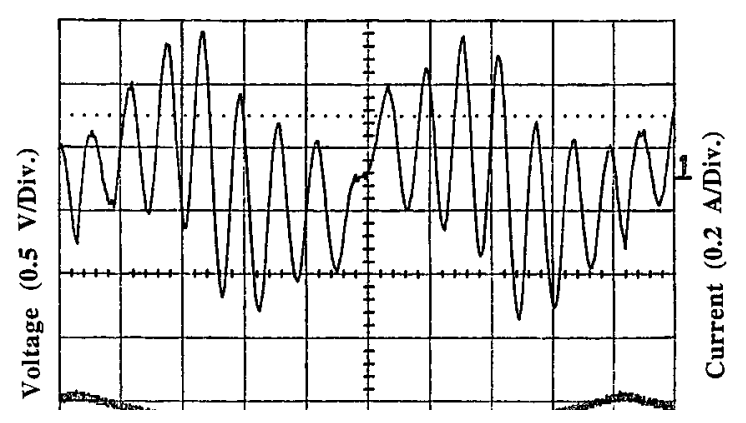

Fig. 3. Wavelength-scanning fringes from the DEFPI. The lower trace in each figure is the driving current applied to the Galvo to tune the wavelength, and the upper trace represents the interference fringes versus the wavelength (a) from the reference cavity with a cavity length of $81.4 \mu \mathrm{m}$ and (b) and (c) from the sensing head with different measured gap lengths.

system's signal-to-noise ratio could be significantly improved.

A set of experimental data is plotted in Fig. 4. The horizontal axis represents the known increments of the sensing length, driven by the translation stage as 


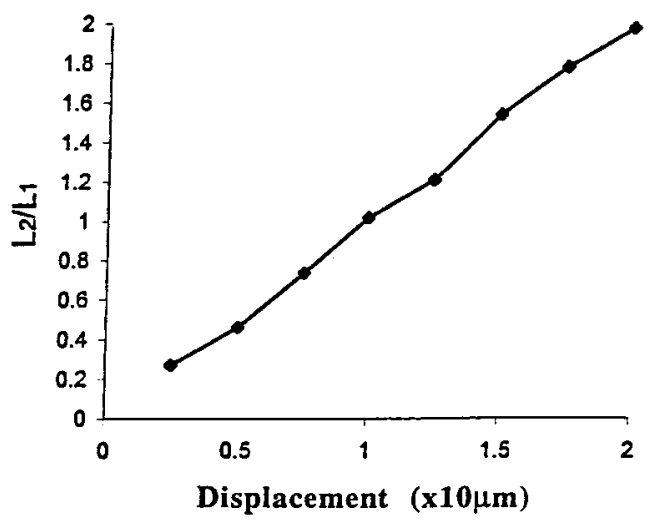

Fig. 4. Experimental plot of the ratio of the sensing-gap length over the reference-cavity length versus the reference-cavity length; measured normalized sensor-cavity length versus the readout from the translation stage. The horizontal axis represents the known increments of the sensing length. The reference-cavity length $L_{1}$ is $81.4 \mu \mathrm{m}$.

shown in Fig. 1. The cavity length $L_{1}$ of the reference sensor was $81.4 \mu \mathrm{m}$. The vertical axis is the ratio of the measured sensing length versus these given increments.

The optical-scanning dual-fiber EFPI (DEFPI) system reported in this paper has been proved to be a simple structure, single-end operation, selfcalibrated, absolute measurement technique with medium accuracy. Since the absolute measurement is obtained based on the comparison with the stable length of the reference cavity, accurate wavelength scanning, which functions only as an interconverter, is not necessary. The sensing scheme described in the paper could thus be used in those applications for which low-cost long-term monitoring is required.

The authors acknowledge the Virginia Center for Innovative Technology for the supporting the research financially.

\section{References}

1. A. D. Kersey, D. A. Jackson, and M. Corko, "A simple fiber Fabry-Perot sensor," Opt. Commun. 45, 71-74 (1983).

2. K. A. Murphy, M. F. Gunther, A. M. Vengsarkar, and R. O. Claus, "Quadrature phase-shifted, extrinsic Fabry-Perot optical fiber sensors," Opt. Lett. 16, 273-275 (1991).

3. T. Li, A. Wang, K. Murphy, and R. Claus, "White-light scanning fiber Michelson interferometer for absolute position-distance measurement," Opt. Lett. 20, 785-787 (1995).

4. C. Belleville and G. Duplain, "White-light interferometric multimode fiber-optic strain sensor," Opt. Lett. 18, 78-80 (1993).

5. B. Fogg, A. Wang, M. Miller, K. Murphy, and R. Claus, "Optical fiber sensor for absolute measurement," in Fiber Optic SensorBased Smart Materials and Structures, R. O. Claus, ed. (Institute of Physics, Bristol, England, 1992), pp. 51-54.

6. D. Wang, Y. Ning, A. Palmer, K. Grattan, and K. Weir, "An optical scanning technique in a white light interferometric system," IEEE Photon. Technol. Lett. 6, 855-857 (1994).

7. G. Ball and W. Morey, "60 mW $1.5 \mu \mathrm{m}$ single-frequency lownoise fiber laser MOPA," IEEE Photon. Technol. Lett. 6, 192194 (1994). 